\title{
Expression of clusterin in pancreatic acinar cell injuries in vivo and in vitro.
}

\author{
Y. Motoo, M.J. Xie, S.B. Su, H. Mouri and N. Sawabu
}

Clusterin is a secretory glycoprotein that is highly induced in several tissues in response to injury. Although anti-apoptotic character is reported in clusterin, pathophysiologic significance of clusterin expression in the pancreas remains largely unknown. The aim of this work was to examine whether clusterin is expressed in spontaneous chronic pancreatitis in the WBN/Kob rat and to investigate the relationship between clusterin and apoptosis in rat pancreatic acinar AR4-2J cells. In the in vivo study, 4-week-old male WBN/Kob rats were sacrificed at every 4 weeks and the pancreatic alterations were studied with pathological and molecular methods. Only male WBN/Kob rats developed chronic pancreatitis at 12 weeks. Pathologically, the peak of inflammation was seen at 12 weeks and those of fibrosis and acinar cell destruction were at 16 weeks. In RT-PCR analysis, clusterin mRNA was expressed at 12 weeks and then decreased. TNF-alpha mRNA peaked at 8 weeks, PAP, IL-6, IL-8 and TGF-beta mRNAs peaked at 12 weeks, and IFN-gamma mRNA peaked at 16 weeks. Fas and FasL mRNAs as well as acinar cell apoptosis assessed by the TUNEL method peaked at 12 and 20 weeks. Immunohistochemistry showed clusterin expression in the cytoplasm of acinar cells. Thus, clusterin may be expressed in pancreatic acinar cells in response to various stresses and in relation to "cytokine/chemokine cascade" in the pancreas. At the onset of chronic pancreatitis, i.e. at 12 weeks, both pro-, and anti-apoptotic factors are expressed in the pancreas of WBN/Kob rats. Acinar cell death might be determined by the balance between these factors. At 20 weeks, expression of only pro-apoptotic factors and insulin-like growth factor (IGF)-I and c-myc mRNAs increased, suggesting that these factors are involved in acinar regeneration and remodeling during the progression of chronic pancreatitis. In the in vitro study, clusterin mRNA and protein were strongly induced in AR4-2J cells treated either with arginine, menadione, TNF-alpha or TGF-beta. In the time course study with arginine or menadione, clusterin mRNA was expressed at 4 hours after the addition of these oxidative stresses and peaked at 24 hours, whereas apoptosis, determined by the DNA fragmentation ELISA assay, peaked at 72 hours. These results show that clusterin is overexpressed in the pancreas at the onset of chronic pancreatitis in vivo and in cultured acinar cells in response to various stimuli in vitro, suggesting that clusterin is a defense mechanism of the exocrine pancreas. 